\title{
Mice Lacking Adrenergic Signaling Have Normal Cochlear Responses and Normal Resistance to Acoustic Injury but Enhanced Susceptibility to Middle-Ear Infection
}

\author{
Stéphane F. Maison ${ }^{1,2,3}$, Mina Le ${ }^{2}$, Erik Larsen ${ }^{2,3}$, Suh-Kyung LeE $^{3}$, John J. Rosowski ${ }^{1,2,3}$, \\ Steven A. Thomas ${ }^{4}$, and M. Charles Liberman ${ }^{1,2,3}$ \\ ${ }^{1}$ Department of Otology and Laryngology, Harvard Medical School, Boston, MA, USA \\ ${ }^{2}$ Eaton-Peabody Laboratory, Massachusetts Eye and Ear Infirmary, 243 Charles St., Boston, MA 02114-3096, USA \\ ${ }^{3}$ Program in Speech and Hearing Biosciences and Technology, Harvard-MIT, Cambridge, MA, USA \\ ${ }^{4}$ Department of Pharmacology, University of Pennsylvania, Philadelphia, PA, USA
}

Received: 24 March 2010; Accepted: 4 May 2010; Online publication: 26 May 2010

\begin{abstract}
The vasculature and neurons of the inner ear receive adrenergic innervation from the cervical sympathetic chain, and adrenergic receptors may be expressed by cells of the organ of Corti and stria vascularis, despite a lack of direct sympathetic innervation. To assess the functional role of adrenergic signaling in the auditory periphery, we studied mice with targeted deletion of the gene for dopamine $\beta$-hydroxylase (DBH), which catalyzes the conversion of dopamine to noradrenaline; thus, these mutant mice have no measurable adrenaline or noradrenaline. $D b h^{-/-}$mice were more susceptible to spontaneous middle-ear infection than their control littermates, consistent with a role for sympathetics in systemic and/or local immune response. At 6-8 weeks of age, cochlear thresholds and suprathreshold responses assessed by auditory brainstem responses and distortion product otoacoustic emissions, as well as light-microscopic morphology, were indistinguishable from controls, if ears with conductive hearing loss were eliminated. $D b h^{-/-}$mice were no more susceptible to acoustic injury than controls, despite prior reports that sympathectomy reduces noise damage. $D b h^{-/-}$mice showed enhancement of shock-evoked olivocochlear suppression of cochlear responses, which may arise from the loss of
\end{abstract}

Correspondence to: Stéphane F. Maison - Eaton-Peabody Laboratory · Massachusetts Eye and Ear Infirmary · 243 Charles St., Boston, MA 02114-3096, USA. Telephone: +1-617-5733745; fax: +1-617-7204408; email: stephane_maison@meei.harvard.edu adrenergic inputs to olivocochlear neurons in the brainstem. However, adrenergic modulation of olivocochlear efferents does not mediate the protective effect of contralateral cochlear destruction on ipsilateral response to acoustic overexposure.

Keywords: dopamine $\beta$-hydroxylase, sympathetic nervous system, otitis media, olivocochlear

\section{INTRODUCTION}

The mammalian auditory periphery receives a rich adrenergic innervation from the sympathetic nervous system, specifically from the stellate and superior cervical ganglia. The mucosa of the middle ear and Eustachian tube receive a network of adrenergic fibers with fine varicosities in close proximity to small-caliber blood vessels (Nagaraj and Linthicum 1998; Uddman et al. 1983). In the inner ear, sympathetic projections include at least two components (Hozawa et al. 1989; Hozawa and Kimura 1990; Spoendlin 1981; Spoendlin and Lichtensteiger 1967): (1) a vessel-associated component, arising from the stellate ganglion bilaterally, and forming a dense plexus around the major arterial supply and (2) a vessel-independent component, arising from the superior cervical ganglion ipsilaterally, and giving rise to numerous terminals in regions of the osseous spiral lamina where axons and cell bodies of the cochlear nerve fibers are found 
(Borg et al. 1974; Densert and Flock 1974; Spoendlin and Lichtensteiger 1966).

Physiological experiments have reported changes in cochlear function upon stimulation or removal of the sympathetic chain, but effects tend to be small and/or inconsistent. Stimulation, or removal, of the stellate ganglion can decrease, or increase (respectively), cochlear blood flow bilaterally (Laurikainen et al. 1997; Laurikainen et al. 1993; Ren et al. 1993a), consistent with a role for the cochlear sympathetics in regulating vascular smooth muscle tone, as in other organ systems. Removal of the superior cervical ganglion has no effect on cochlear blood flow (Laurikainen et al. 1997), but several labs have shown that this type of sympathectomy renders the ear less vulnerable to acoustic injury (Borg 1982; Hildesheimer et al. 2002; Horner et al. 2001). Paradoxically, others have shown that electrical stimulation of the superior cervical ganglion also decreases noise-induced threshold shift (Wada et al. 1999). Shocking the sympathetic chain can also change the amplitude (Pickles 1979) or latency (Lee and Møller 1985) of cochlear neural potentials.

Although hearing researchers have manipulated the cervical sympathetic chain to study sympathetic modulation of inner ear function, adrenergic signaling in the auditory periphery may arise from other pathways. Firstly, recent immunostaining and/or RTPCR evidence suggests that adrenergic receptors are expressed by hair cells, the stria vascularis, and the spiral ligament (Fauser et al. 2004; Schimanski et al. 2001), despite the fact that projections from the sympathetic ganglia do not reach these structures (Densert 1974; Hozawa et al. 1989; Shibamori et al. 1994; Spoendlin and Lichtensteiger 1966). Pharmacological evidence for adrenergic modulation of $\mathrm{K}^{+}$ secretion in the stria via $\beta_{1}$ receptors has further strengthened the idea that circulating, or locally synthesized, catecholamines may also be important in cochlear function and dysfunction (Schimanski et al. 2001). Secondly, in addition to direct inner ear effects via adrenergic projections from the peripheral sympathetic chain to the cochlea, central adrenergic projections from the locus coeruleus to the olivocochlear (OC) efferent system (Mulders and Robertson 2005a, b; Woods and Azeredo 1999) may also modulate cochlear function, or responses to stress, indirectly by modulating the strength of this soundevoked feedback pathway. Indeed, the peculiar phenomenon whereby destruction of the contralateral cochlea protects the ipsilateral ear from subsequent acoustic overstimulation (Rajan and Johnstone 1989) could arise from "stress"-induced activation of the olivocochlear reflex via its central adrenergic inputs.

To test this hypothesis, and to revisit the functional role of the cochlear sympathetics in particular, and of adrenergic signaling in the auditory periphery more generally, we studied mice with targeted deletion of the gene for dopamine $\beta$-hydroxylase (DBH), the enzyme in the catecholamine biosynthetic pathway that converts dopamine to norepinephrine (Thomas et al. 1995). These mutant mice do not survive gestation without dihydroxyphenylserine in the maternal drinking water, a precursor that can be converted to noradrenaline in the absence of DBH. However, after birth, the $D b h^{-/-}$mice are viable, without special dietary supplements, despite complete absence of noradrenaline and adrenaline. We found the $\mathrm{Dbh}^{-/-}$ mice to be significantly more vulnerable to middle-ear infection than littermate controls. However, once ears with middle-ear disease were removed from consideration, we found no evidence for cochlear dysfunction, no evidence for altered vulnerability to acoustic overstimulation and clear evidence that the protective effects of contralateral cochlear destruction do not require adrenergic signaling.

\section{MATERIALS AND METHODS}

\section{Mutant mouse line and general procedures}

Mice in this study were 6-8-week-old hybrids of 129/ SvCPJ and C57BL/6J strains. The deletion of the gene for DBH is described elsewhere (Thomas et al. 1995). Most $D b h^{-/-}$mice die in utero, unless a catecholamine precursor is supplied in the maternal drinking water (Thomas et al. 1995). After birth, $D b h^{-/-}$mice are viable, despite the complete absence of measurable epinephrine or norepinephrine, with no other overt phenotype than ptosis (Thomas et al. 1998). These mutants have altered metabolism, thermoregulation, cardiovascular tone, maternal behavior and deficits in motor function, learning and memory (Thomas and Palmiter 1997a, b, c). Heterozygous mice $\left(D b h^{+/-}\right)$ have normal catecholamine levels (Thomas et al. 1998) and thus were used as controls. The animal care committee of the Infirmary approved the care and use of the animals. For terminal procedures, mice were anesthetized with urethane (1.2 g/kg i.p.); for survival procedures, mice were anesthetized with pentobarbital (71.2 mg/kg i.p.). All physiological measurements were conducted in a temperaturecontrolled soundproof chamber maintained at $\sim 32^{\circ} \mathrm{C}$.

\section{Middle-ear motion: laser-doppler vibrometry}

After anesthetization, the cartilaginous external ear canal was removed to expose the pars tensa and pars flaccida of the tympanic membrane. Ossicular motion, i.e., umbo velocity, in response to pure tones swept from 1 to $50 \mathrm{kHz}$ was measured using a laser-Doppler 
vibrometer. A small (20-50 $\mu \mathrm{m}$ diameter) reflective bead was placed on the umbo. An open-backed coupler attached to an earphone (Beyerdynamic DT48) by an $8 \mathrm{~cm}$ long gum-rubber tube was sealed over the ear-canal opening, and the beam of a Polytec laser vibrometer was focused on the bead. The output of the vibrometer was averaged synchronously during repetitive acoustic stimulation with broad-band ( 0.5 to $50 \mathrm{kHz})$ chirps $(n=500-2,000)$. The amplitude of the chirps was between 80 to $100 \mathrm{~dB}$ SPL. After motion measurement, a probe-tube coupled to a one-fourth inch condenser microphone was placed in the coupler to measure the ear-canal sound pressure. An independently determined probe-tube transfer function was used to convert the measured microphone voltage to sound pressure, and the umbo-velocity transfer function was computed as the ratio of the measured velocity and ear-canal pressure (Rosowski et al. 2003).

\section{Cochlear responses: ABRs and DPOAEs}

For measurement of auditory brainstem responses (ABRs) and distortion product otoacoustic emissions (DPOAEs), a small slit was made in the ear canal to allow microscopic evaluation of the condition of the tympanic membrane. Acoustic stimuli were delivered using a custom acoustic assembly consisting of two electrostatic earphones (EC-1, Tucker Davis Technologies) to generate primary tones and a Knowles miniature microphone (EK-3103) to record ear-canal sound pressure. Stimuli were generated digitally (National Instruments, digital I-O board 6052E). Earcanal sound pressure and electrode voltage were amplified and digitally sampled at $20 \mu$ s for analysis of response amplitudes (National Instruments, digital I-O board 6052E).

For ABRs, needle electrodes were inserted at vertex and pinna, with a ground electrode near the tail. Stimuli were 5 -ms tone pips $\left(0.5\right.$-ms rise-fall with a $\cos ^{2}$ onset, delivered at $35 / \mathrm{s})$. The response was amplified $(10,000 \times)$, filtered $(100 \mathrm{~Hz}-3 \mathrm{kHz})$, digitized and averaged in a LabVIEW-driven data-acquisition system. Sound level was raised in $5 \mathrm{~dB}$ steps from $10 \mathrm{~dB}$ below threshold up to $80 \mathrm{~dB}$ SPL. At each sound level, 1,024 responses were averaged (with stimulus polarity alternated), using an "artifact reject" whereby response waveforms were discarded when peak-topeak amplitude exceeded $15 \mathrm{~V}$. Upon visual inspection of stacked waveforms, "threshold" was defined as the lowest SPL level at which any wave could be detected, usually corresponding to the level step just below that at which the peak-to-peak response amplitude rose significantly above the noise floor $(\sim 0.25 \mu \mathrm{V})$. For amplitude-vs.-level functions, the wave 1 peak was identified by visual inspection at each sound level and the peak-to-peak amplitude computed.

For measurement of DPOAEs at $2 f_{1}-f_{2}$ the primarytone frequency ratio, $\left(f_{2} / f_{1}\right)$, was 1.2 and $f_{2}$ level was $10 \mathrm{~dB}$ below $f_{1}$ level. For each $f_{2} / f_{1}$ primary pair, levels were swept in $5 \mathrm{~dB}$ steps from $20 \mathrm{~dB}$ SPL to $80 \mathrm{~dB}$ SPL (for $f_{2}$ ). At each level, both waveform and spectral averaging were used to increase the signal to noise ratio of the recorded ear-canal sound pressure, and the amplitude of the DPOAE at $2 f_{1}-f_{2}$ was extracted from the averaged spectra, along with the noise floor at nearby points in the spectrum. Iso-response curves were interpolated from plots of DPOAE amplitude-vs.sound level. "Threshold' was defined as the $f_{1}$ level required to produce a DPOAE at $0 \mathrm{~dB}$ SPL.

\section{Medial olivocochlear assay}

A posterior craniotomy and partial cerebellar aspiration were performed to expose the floor of the IVth ventricle. To stimulate the olivocochlear bundle, shocks (monophasic pulses, $150 \mu$ s duration, 200/s) were applied through fine silver wires $(0.4 \mathrm{~mm}$ spacing) placed along the midline, spanning the olivocochlear decussation. Shock threshold for facial twitches was determined, muscle paralysis induced with $\alpha$-D-tubocurarine $(1.25 \mathrm{mg} / \mathrm{kg}$ i.p. $)$, and the animal connected to a respirator via a tracheal cannula. Shock levels were raised to $6 \mathrm{~dB}$ above twitch threshold. During the olivocochlear suppression assay, $f_{2}$ level was set to produce a DPOAE $10-15 \mathrm{~dB}$ above the noise floor. To measure olivocochlear effects, repeated measures of baseline DPOAE amplitude were first obtained $(n=54)$, followed by a series of 70 contiguous periods in which DPOAE amplitudes were measured with simultaneous shocks to the olivocochlear bundle and additional periods during which DPOAE measures continued after the termination of the shock train. During paralysis, heart rate was monitored, and urethane boosters were administered every hour at $25 \%$ of the initial dose $(0.3 \mathrm{~g} / \mathrm{kg}$ i.p. $)$

\section{Acoustic overexposure}

For studies of permanent acoustic injury, animals were exposed to free-field sound, while awake and unrestrained, in a small reverberant chamber for $2 \mathrm{~h}$ to an $8-16 \mathrm{kHz}$ octave band noise presented at $100 \mathrm{~dB}$ SPL. The exposure stimulus was generated by a custom white-noise source, filtered (Brickwall Filter with a $60 \mathrm{~dB}$ /octave slope), amplified (Crown power amplifier), and delivered (JBL compression driver) through an exponential horn fitted securely to a hole in the top of a reverberant box. Sound exposure levels were measured at four positions within each cage using a $0.25^{\prime \prime}$ Bruel and Kjaer condenser microphone: sound 
pressure varied by less than $0.5 \mathrm{~dB}$ across these measurement positions.

To study protective effects of contralateral cochlear destruction on temporary threshold shifts, groups of $D b h^{-/}$and controls were anesthetized and exposed, via a closed acoustic system inserted in the ear canal, to an $11-\mathrm{kHz}$ tone at $102 \mathrm{~dB}$ SPL for $1 \mathrm{~min}$. DPOAE thresholds were measured before and immediately following acoustic overexposure. Twenty-four hours before the exposure, some animals from each genotype underwent contralateral cochlear destruction under general anesthesia (pentobarbital, $71.2 \mathrm{mg} / \mathrm{kg}$ i.p.) by removing the tympanic membrane and ossicles and destroying the cochlea by penetrating the cochlear capsule with a right-angle microprobe.

\section{Cochlear processing and immunostaining}

To study cochlear histopathology in $\mathrm{Dbh}^{-/-}$, mutants and controls were perfused intracardially with $2.5 \%$ glutaraldehyde and $1.5 \%$ paraformaldehyde in phosphate buffer. Cochleas were decalcified in EDTA, osmicated and dehydrated in ethanols and propylene oxide, embedded in araldite resins, and sectioned at $40 \mu \mathrm{m}$ on a Historange with a carbide steel knife. Sections were mounted on slides and coverslipped.

To study the normal adrenergic innervation of the inner ear, mice were fixed by intravascular perfusion of $4 \%$ paraformaldehyde with $0.1 \%$ glutaraldehyde in PBS. Cochleas were decalcified, dissected into halfturns and then incubated in 5\% normal horse serum with 2\% Triton X-100 in PBS for $1 \mathrm{~h}$. This was followed by overnight incubation in primary antibody(s): (1) rabbit anti-DBH from Abcam at 1:200, and/or (2) sheep anti-tyrosine hydroxylase (TH) from Calbiochem at 1:2,000, and/or (3) chicken anti-heavy neurofilament from Chemicon at 1:10,000, followed by appropriate secondary antibodies for 90 min: (1) biotinylated donkey anti-rabbit from Jackson ImmunoResearch Laboratories followed by streptavidin-coupled Alexafluor 568 from Molecular Probes; and/or (2 and 3) Alexafluor-488 chicken anti-goat from Molecular Probes or Alexafluor-488 coupled goat anti-chicken, all at 1:1,000.

\section{RESULTS}

$\mathrm{DBH}$ immunostaining and the adrenergic innervation of the normal inner ear

The adrenergic innervation of the inner ear has been studied by either histochemistry (i.e., Falck-Hillarp), or by immunohistochemistry for DBH and/or TH, in several mammalian species including cat (Spoendlin and Lichtensteiger 1966), rabbit (Densert 1974), guinea pig (Hozawa et al. 1989), rat (Shibamori et al. 1994) and monkey (Hozawa and Kimura 1990). Here, we used immunostaining to verify that the same general patterns are seen in the mouse. Specifically, we observed co-localization of anti-TH and anti-DBH immunostaining in a diffuse plexus of thin, varicose, presumably unmyelinated fibers distributed throughout the modiolus, the spiral ganglion and the osseous spiral lamina (Fig. 1A, B, C). Consistent with prior reports (Darrow et al. 2006; Hozawa et al. 1989; Hozawa and Kimura 1990), DBH-positive fibers in mouse were never seen within the organ of Corti. In other species, it has been demonstrated that virtually all these thin adrenergic fibers originate from the ipsilateral superior cervical ganglion (Densert 1974; Shibamori et al. 1994).

In the osseous spiral lamina, these sympathetic fibers run among the more numerous myelinated peripheral axons of the cochlear nerve fibers: the cochlear sensory axons immunostain for high molecular weight neurofilament; the sympathetics do not (Fig. 1A). Many of these sympathetic fibers end blindly at the peripheral edge of the osseous spiral lamina, in the region of the habenula perforata, where the cochlear sensory fibers lose their myelin sheaths (open arrows in Fig. 1C). Ultrastructural studies in other mammals have shown intimate contact between sympathetic terminals and cochlear nerve dendrites in this region (Densert and Flock 1974). Other sympathetics appear to spiral along the vessels of the tympanic lip (e.g., filled arrow in Fig 1C). The photomicrographs in Figure 1 are from the middle of the cochlea: the density of this adrenergic innervation appeared roughly constant from apex to base along the cochlear spiral.

\section{Middle-ear infection and conductive hearing loss} in $D b h^{-1-}$ mice

Signs of active or resolved middle-ear infection are often visible by microscopic inspection of the tympanic membrane (TM): redness is a sign of acute, or early-stage, infection, and opacity is a sign of prior, or chronic, infection (Rosowski et al. 2003). As shown in Figure 2B, 26/64 (41\%) of $D b h^{-/-}$mice showed a cloudy TM (grey bars in histograms) during preparation for DPOAE/ABR recordings vs. only 4/56 (7\%) of control animals (Fig. 2A), suggesting a roughly 6fold increase in susceptibility to middle-ear infection in the absence of adrenergic signaling.

Given that DPOAEs are created within the cochlea by sound delivered through the middle ear, and then propagated back through the ossicles to the ear canal, DPOAE thresholds are more sensitive to middle-ear disease than ABR thresholds (Qin et al. 2010): for DPOAEs, the sounds have to traverse the middle ear twice. As shown in Figure 2, B, the distribution of 

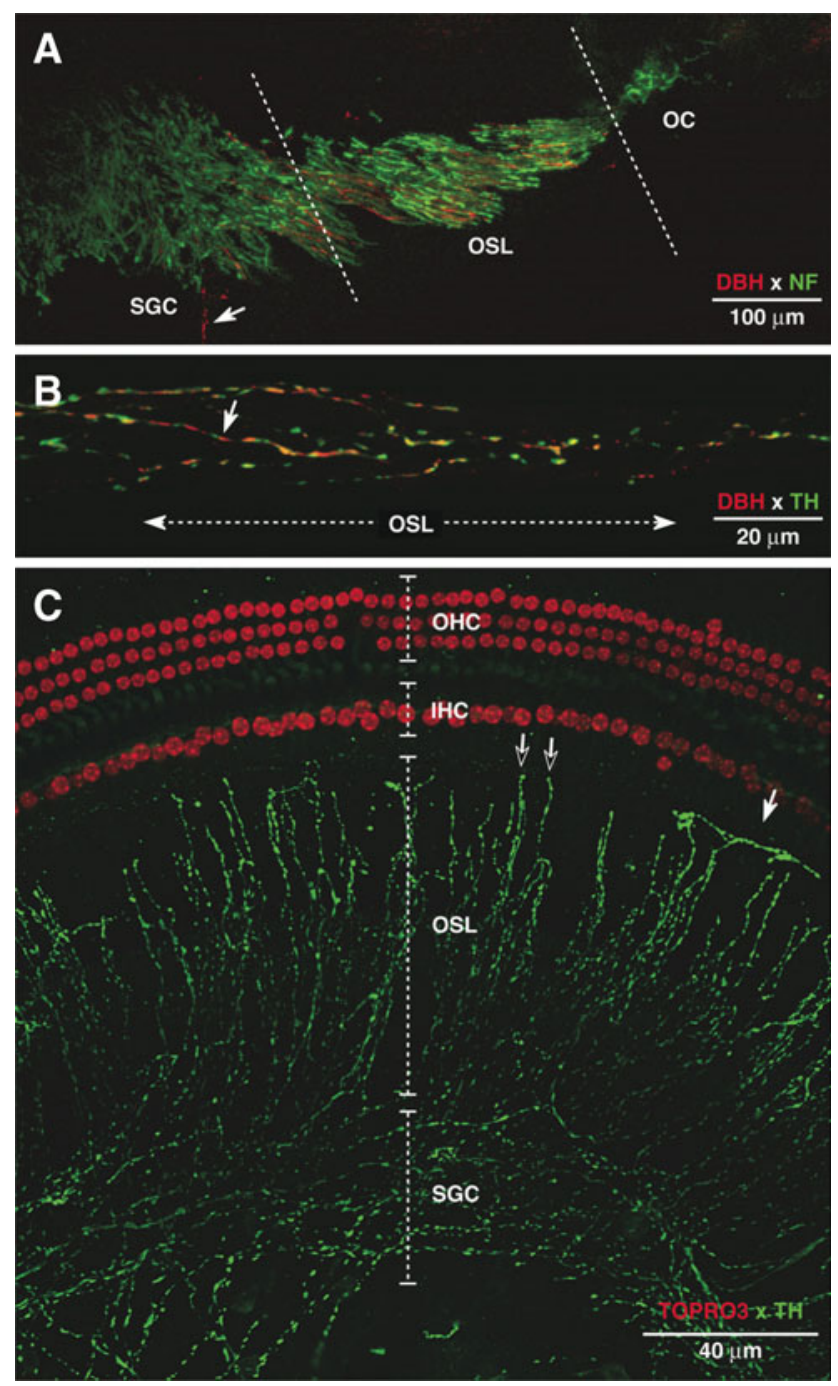

FIG. 1. Immunostaining for $\mathrm{DBH}$ and/or $\mathrm{TH}$ reveals the profuse adrenergic innervation of the inner ear in a normal mouse. A Crosssection through the upper basal turn shows DBH-positive fibers (red) in the spiral ganglion cell (SGC) region and in the osseous spiral lamina (OSL), but not in the organ of Corti (OC). Doubleimmunostaining shows that the small, unmyelinated $\mathrm{DBH}$-positive fibers (e.g., arrow) do not co-localize with the high molecular weight neurofilaments (NF) seen in the large myelinated cochlear nerve fibers (green). B cross-section through the upper basal turn shows colocalization of $\mathrm{DBH}$ (red) and TH (green) in all thin varicose fibers in the OSL. TH and DBH immunostaining often alternate in adjacent regions of the same fiber (arrow). $\mathbf{C}$ surface view of the upper basal turn shows the profuse sympathetic innervation (green) in the OSL, with many fibers ending blindly near the habenula (open arrows) or spiraling at the tympanic lip (filled arrow). For clarity, nuclear staining with TOPRO3 (red) has been erased in the OSL region.

DPOAE thresholds in the present study was bimodal, and, indeed, all the ears with obvious middle-ear infection, i.e., cloudy TM, were in the high-threshold group $(<60 \mathrm{~dB}$ at $16 \mathrm{kHz})$. The DPOAE thresholds, and signs of middle-ear disease, were often asymmetrical: some animals had excellent thresholds (and a clear TM) in one ear, yet poor thresholds (and a cloudy TM) in the other.
To test the idea that the ears with clear TM and greatly elevated DPOAE threshold (Fig. 2A, B) also had middle-ear disease, we selected a group (four animals, eight ears) with a range of DPOAE thresholds for direct measurement of ossicular motion and histological analysis of middle-ear pathology. Middle-ear motion measurements were successful in six ears (one animal died during testing). As shown in Figure 2C, ossicular motion was indeed greatly reduced in two out of three ears with high-threshold DPOAEs, whereas motion was normal in three out of three animals with DPOAE thresholds in the low-threshold peak. The histological correlations were even more compelling. All five ears with normal DPOAE thresholds had a clean middle ear and a normal round-window membrane (e.g., Fig. 3A). In contrast, all three ears with high DPOAE thresholds had significant cellular or fibrous debris in the middle ear, especially in the round-window niche, and a significant thickening of the round-window membrane (Fig. 3B, C).

Based on these observations, we assume that all the animals in the high-threshold DPOAE peak, regardless of genotype, had acute or chronic middle-ear infections at the time of testing. By this definition, the frequency of middle-ear disease was $52 \%$ in $D b h^{-/-}$ ears (33/64: Fig. 2B) vs. $16 \%$ in controls $(9 / 56$ : Fig. 2A). Therefore, to study possible cochlear phenotypes, we removed these high-threshold ears from further consideration.

\section{Cochlear responses and morphology in $\mathrm{Dbh}^{-/-}$ mice}

With putative middle-ear pathology eliminated, absence of adrenergic signaling has little impact on cochlear thresholds or suprathreshold responses, as measured by either ABRs or DPOAEs (Fig. 4). Indeed, ABR thresholds (Fig. 4A), Wave 1 amplitudes (Fig. 4C) and latencies (Fig. 4E) were statistically indistinguishable from controls at all test frequencies. DPOAE thresholds (Fig. 4B) and amplitude-vs.-level functions (Fig. 4D) were identical to controls at the higher frequencies. At the lower test frequencies $\left(f_{2}=8.0\right.$ or $\left.11.3 \mathrm{kHz}\right)$ a small (10 dB) but highly significant $(p<0.001$ by unpaired Student's $t$ test) threshold elevation was observed in $D b h^{-/-}$mice. This elevation, present in DPOAEs and absent in ABRs, likely reflects conductive dysfunction (Qin et al. 2010), perhaps from decreased middle-ear compliance due to modest fluid accumulation or negative pressure developing in the middle-ear space.

Cochlear morphology in $\mathrm{Dbh}^{-/-}$mice was assessed by light-microscopic evaluation of plastic sections of aldehyde-fixed and osmium post-fixed cochleas (Fig. 5). No systematic abnormalities were seen in 

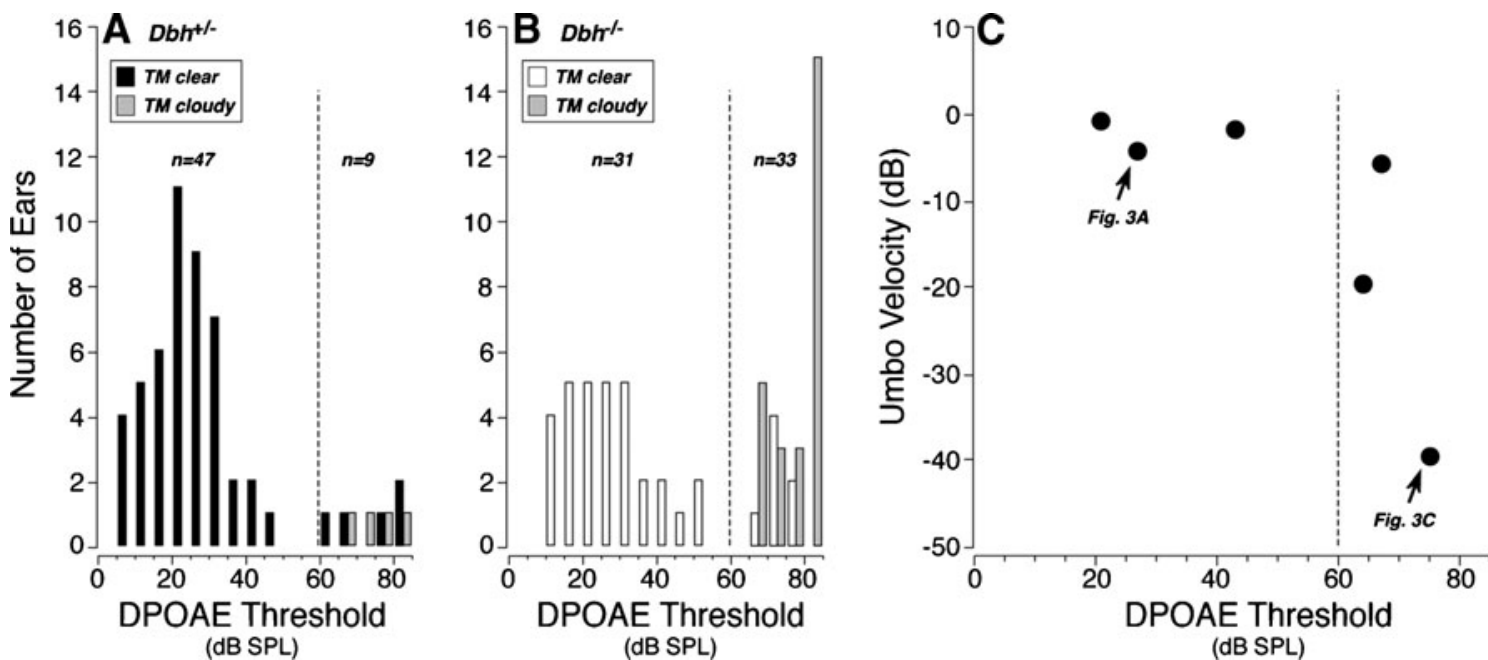

FIG. 2. $D b h^{-1-}$ mice are more susceptible to middle-ear infections and conductive hearing loss than their heterozygous counterparts. In both $D b h^{+/-}$(A) and $D b h^{-/-}$mice (B), the distribution of DPOAE thresholds (for $f_{2}=16 \mathrm{kHz}$ ) was bimodal, and all ears with a cloudy tympanic membrane (TM; $n=4$ and $n=26$, respectively) fell in the high-threshold peak. For each genotype, the number of ears with low vs. high thresholds (re $60 \mathrm{~dB}$ SPL) is given. C Umbo-velocity

any structure of the inner ear, including hair cells and spiral ganglion cells, stria vascularis, spiral ligament, and all supporting structures of the cochlear duct. For each case evaluated, serial sections through the entire cochlea were examined. Special attention was paid to the basal turn hair cells and to the type-IV fibrocytes in the spiral ligament, which in the mouse ear are the most vulnerable elements to acoustic injury (Wang et al. 2002) or age-related degenerative changes (Hequembourg and Liberman 2001).

\section{Olivocochlear effects in $D b h^{-/-}$mice}

Medial olivocochlear fibers project to outer hair cells and form the effector arm of a negative feedback pathway (for review, see Guinan 1996). When activated by sound or electrical stimulation, cochlear measurements were made in a selected group of $D b h^{+/+}$and $D b h^{+/-}$ mice with various DPOAE thresholds but normal-appearing TMs: the reduced ossicular motion in the high-threshold cases suggests that the threshold shift is of conductive origin. Umbo velocity is shown here at $11 \mathrm{kHz}$, the frequency near $2 f_{1}-f_{2}$ when the $f_{2}$ frequency is $16 \mathrm{kHz}$, as for panels $\mathbf{A}$ and $\mathbf{B}$. Photomicrographs of one low- and one high-threshold ear (arrows) appear in Figure 3.

efferent neurons decrease the normal contribution of outer hair cells to cochlear mechanical amplification, and, as a consequence, DPOAE amplitudes are suppressed. This suppression provides a sensitive measure of olivocochlear function. Noting that olivocochlear efferent neurons are the target of noradrenergic inputs (Mulders and Robertson 2005a, b; Woods and Azeredo 1999), we investigated whether the lack of adrenergic signaling affects olivocochlear efferent function.

DPOAE amplitudes evoked by low-level tones were measured before, during and after delivering a 70 -sec shock train to the olivocochlear bundle at the floor of the IVth ventricle. Figure $6 \mathrm{~A}$ shows average runs measured at $\mathrm{f}_{2}=22.6 \mathrm{kHz}$. Qualitatively, olivocochlear effects were normal in $\mathrm{Dbh^{-1- }}$ mice: (1) at shock-train onset, DPOAE amplitudes were suppressed (Fig. 6A,

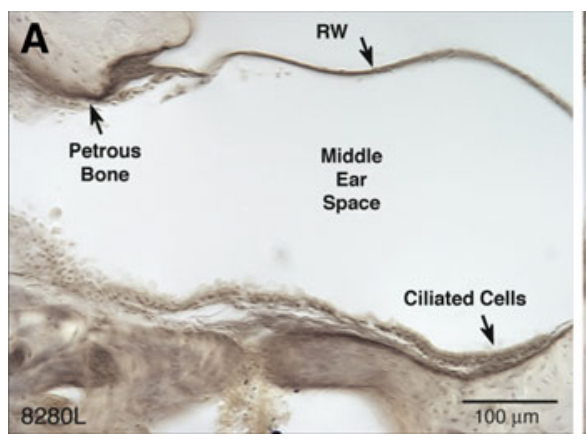

FIG. 3. When mice show high DPOAE thresholds, despite a normal-appearing TM, histological analysis shows cellular debris and/or fibrous tissue in the round-window niche. A Normal roundwindow niche from an ear with excellent DPOAE thresholds $(27 \mathrm{~dB}$ $\mathrm{SPL}$ at $16 \mathrm{kHz}$ ). B and $\mathbf{C}$ Pathological appearance of the round-

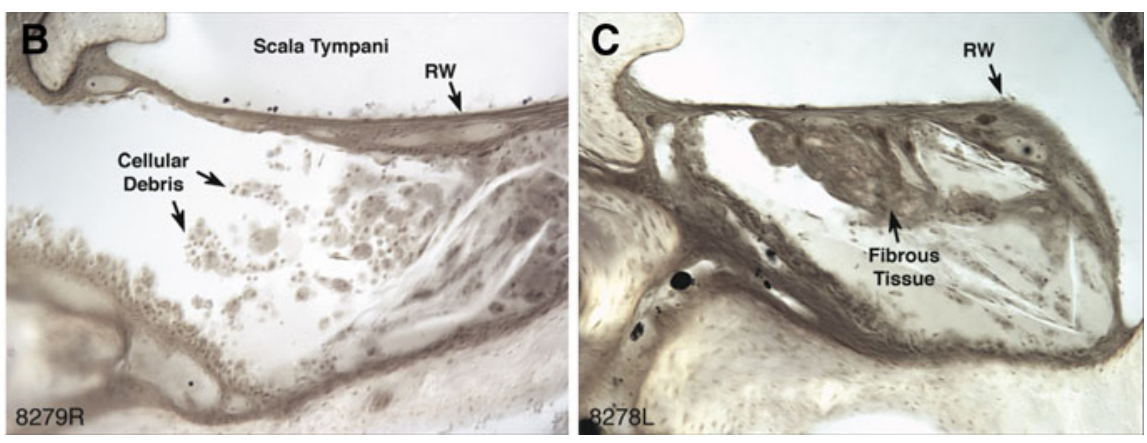

window niche in two cases with elevated DPOAE thresholds (64 and $75 \mathrm{~dB} \mathrm{SPL}$, respectively, at $16 \mathrm{kHz}$ ). All three ears were from a selected group of $D b h^{+/+}$and $D b h^{+/-}$mice. The tissue orientation in panel $\mathbf{C}$ is different: the round-window niche communicates with the rest of the middle ear in another section. 

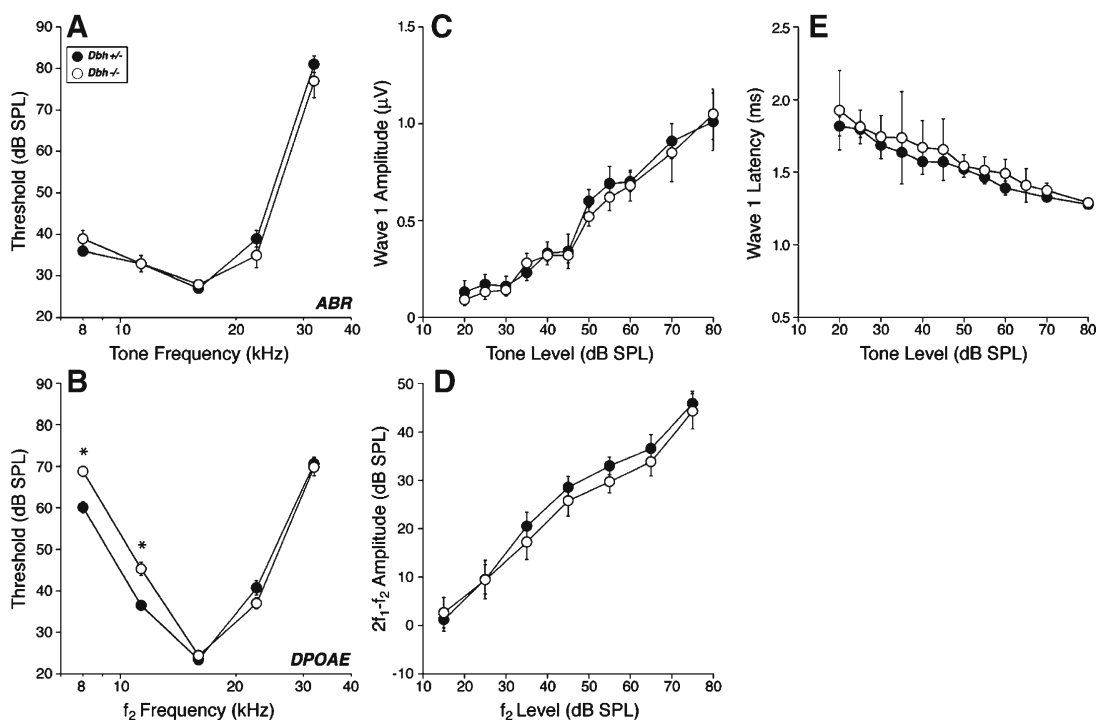

FIG. 4. If ears with demonstrable or putative middle-ear disease are ignored (see Fig. 1), lack of DBH produces only subtle changes in cochlear thresholds $(\mathbf{A}, \mathbf{B})$ suprathreshold responses $(\mathbf{C}, \mathbf{D})$, or neural latencies (E). A, B Mean thresholds $\left( \pm\right.$ SEM) for 6-8 weeks $D b h^{-1-}$ and $D b h^{+/-}$ears by ABRs or DPOAEs. ABR thresholds are statistically indistinguishable between the genotypes; DPOAE threshold differences are significant at 8 and $11.3 \mathrm{kHz}(p<0.001$ by $t$ test). C, D

"during shocks" window); (2) this suppression was maximal for tones at $16-22 \mathrm{kHz}$ reflecting the innervation density of olivocochlear terminals along the cochlear spiral in mouse (Maison et al. 2003a); 3) suppression decayed during the 70-s shock train as is observed in auditory nerve response (Wiederhold and Kiang 1970); and (4) a slow enhancement of DPOAEs (Maison et al. 2007) was usually visible after termination of the shock train (Fig. 6A, "post-shocks" window). Quantitatively, there was an increase in the mean magnitude of efferent-mediated cochlear suppression (Fig. 6B), which was significant ( $p=$ 0.003 by two-way ANOVA). The small changes in magnitude of post-shock enhancements were not statistically significant.

\section{Resistance to acoustic injury in $D b h^{-/-}$mice}

Prior studies have shown that surgical sympathectomy can decrease noise-induced threshold shift, both temporary (Hildesheimer et al. 1991; Horner et al. 2001) and permanent (Borg 1982; Hildesheimer et al. 2002), possibly by eliminating a noise-evoked decrease in cochlear blood flow mediated by sympathetic innervation of the cochlear vasculature. However, there is also strong evidence for adrenergic receptors in the stria vascularis and the spiral ligament (Schimanski et al. 2001), which are nowhere near a sympathetic innervation, and therefore putatively responsive to systemic epinephrine and/or locally produced norepinephrine. Thus, to assess the
Mean amplitude-vs.-level functions from the same ears in $\mathbf{A}$ and $\mathbf{B}$, as seen via ABRs (Wave 1) or DPOAEs, evoked by tones (ABR) or with $f_{2}$ (DPOAEs) at $16 \mathrm{kHz}$. E Mean latencies $( \pm$ SEM) for Wave 1 of the ABR evoked at $16 \mathrm{kHz}$. For all these panels, group sizes for DPOAEs $\left(D b h^{-1-} n=84 ; D b h^{+-} n=46\right)$ are larger than those for ABR $\left(D b h^{-1-}\right.$ $n=19 ; D b h^{+/} n=37$ ), because DPOAEs were usually recorded from both ears, whereas ABRs were recorded from only one side.

vulnerability of noise-stressed ears in animals with a complete lack of both neural and humoral adrenergic signaling, we exposed matched groups of transgenic mice to a noise band designed to produce a permanent threshold shift, as measured 1-week post-exposure. As shown in Figure 7, the noise vulnerability of $D b h^{-/-}$mice was virtually identical to that of littermate controls, whether measured by ABR or DPOAE metrics.

Adrenergic signaling can excite the olivocochlear neurons via inputs from the locus coeruleus to olivocochlear cells in the brainstem (Wang and Robertson 1997; Mulders and Robertson 2005a), and sound-evoked activation of the olivocochlear pathway can protect the ear from acoustic injury (Maison et al. 2002). These observations suggested that adrenergic excitation of the olivocochlear pathway might be involved in the phenomenon whereby destruction or silencing of the contralateral ear prior to ipsilateral overexposure significantly reduces noise-induced threshold shift (Rajan and Johnstone 1989; Robertson and Anderson 1994), but only if the olivocochlear bundle is intact. To test this idea, we first reproduced, in control mice, the protective effects of contralateral cochlear destruction (Fig. 8A), previously reported in guinea pigs (Rajan and Johnstone 1989; Robertson and Anderson 1994). Second, we showed that the protective effect was at least as robust in our $\mathrm{Dbh}^{-/-}$mice, completely lacking all adrenergic signaling (Fig. 8B). Note, as well, that the magnitude of temporary threshold shift induced by 


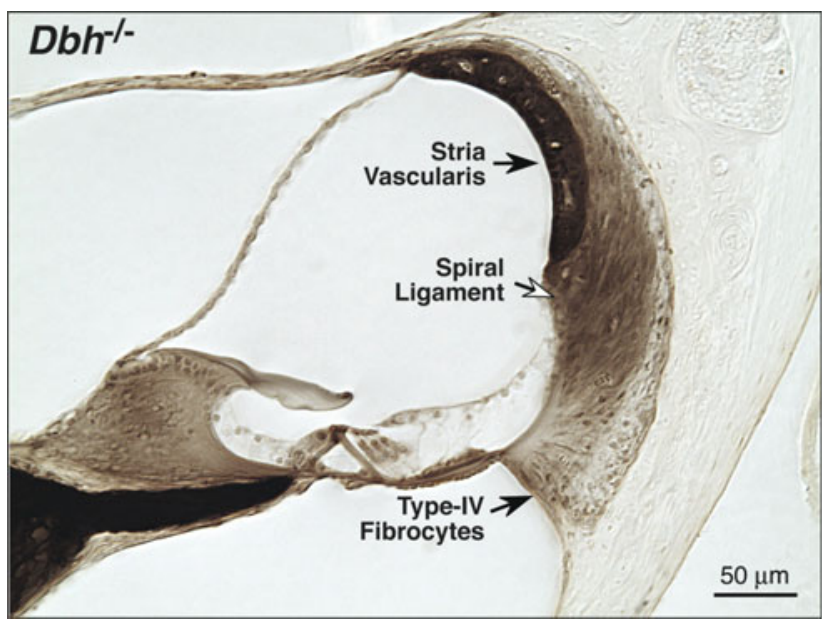

FIG. 5. All structures of the cochlear duct are histologically normal in $\mathrm{Dbh}^{-/-}$mice, as seen in this light micrograph of osmium-stained plastic sections through the upper basal turn.

the pure-tone exposure (in the absence of contralateral cochlear destruction) is also unchanged in the $D b h^{-1-}$ mice.

\section{DISCUSSION}

Immune function in $\mathrm{Dbh}^{-/-}$mice and the prevalence of middle ear infection

The increased prevalence of middle-ear infections in $D b h^{-1-}$ mice (Figs. 2 and 3) may arise from systemic compromise to the immune system. The sympathetic system is a primary pathway for neural regulation of immune function (Hori et al. 1995; Molina 2005).
Primary and secondary immune structures, such as the bone marrow, thymus, spleen, lymph nodes and mucosal tissues, receive a sympathetic innervation (Nance and Sanders 2007). Sympathetic neurons can modulate the expression of cytokines and antibodies by immuno-competent cells (Bellinger et al. 2008; Nance and Sanders 2007), and chemical or surgical sympathectomy can depress the systemic immunological response to infection (Straub et al. 2005; Templeton et al. 2008). Indeed, the $\mathrm{Dbh}^{-/-}$mice studied here have increased susceptibility to infection, T-cell-mediated immunity impairment (Alaniz et al. 1999) along with increased apoptosis of hepatic natural killer cells (Li et al. 2004).

In addition to attenuation of the systemic immune responses, loss of DBH may lead to deficiencies in the local mucosal immune response. The mucosal epithelium of both middle ear and Eustachian tube receive a rich network of adrenergic fibers (Nagaraj and Linthicum 1998; Uddman et al. 1983; Wang et al. 1994), whose normal immunological functions could be disrupted in the $D b h^{-/-}$mice. Another contributing factor may be the loss of adrenergic modulation of Eustachian tube function, as $\alpha$-adrenergic receptor agonists can improve Eustachian tubal patency (Svane-Knudsen et al. 1986), and failure to properly aerate the middle-ear space is thought to be important in the genesis of some middle-ear infections.

Effects of poor middle-ear aeration include negative middle-ear pressure and fluid accumulation, and either the pressure or middle-ear fluid may have caused the slight elevation of cochlear thresholds seen
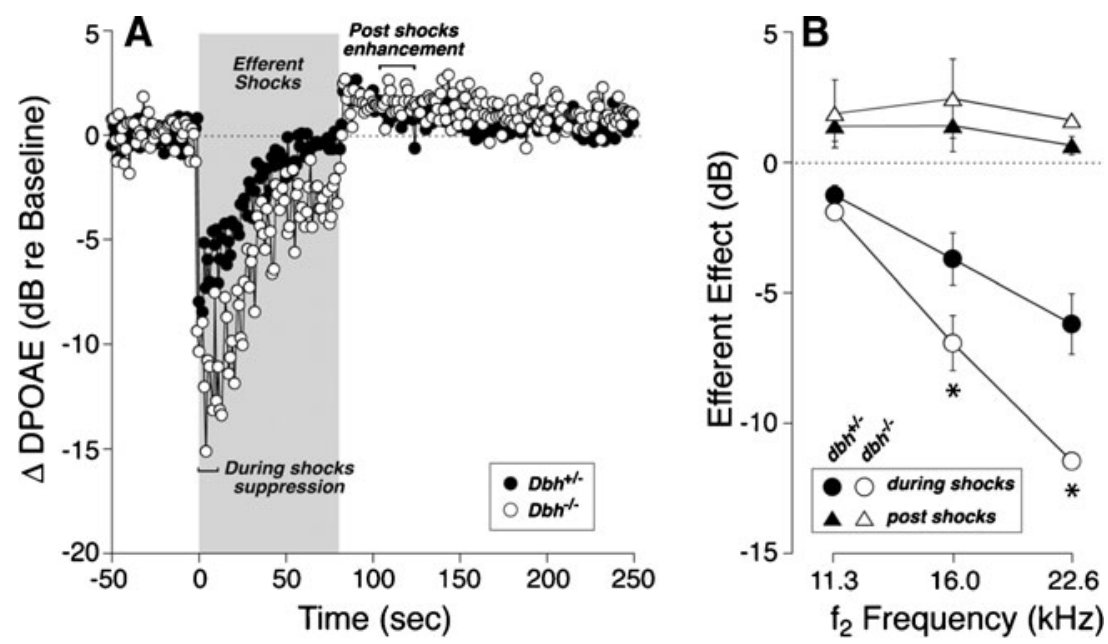

FIG. 6. Efferent-mediated suppression of cochlear responses is enhanced in $D b h^{-1-}$ mice. To assay efferent effects, DPOAE amplitudes evoked by low-level tones were measured before, during and after delivering a 70-s shock train to the olivocochlear bundle at the floor of the IVth ventricle. A Amplitude of DPOAE is plotted as deviation from the mean value seen before the shock-train onset: i.e., the difference in $\mathrm{dB}$ between the measured DPOAE (in $\mathrm{dB} S \mathrm{SPL}$ ) and the mean amplitude (in $\mathrm{dB} S P L)$ of the pre-shocks DPOAE. Data are means of all runs from $D b h^{+/-}(n=5)$ and $D b h^{-/-}(n=3)$ for $f_{2}$ at $22.6 \mathrm{kHz}$. B Mean suppressive (during shocks) and enhancing (post-shocks) effects of efferent stimulation as a function of stimulus frequency. Time periods for measurement of these effects are indicated by the brackets in $\mathbf{A}$. 

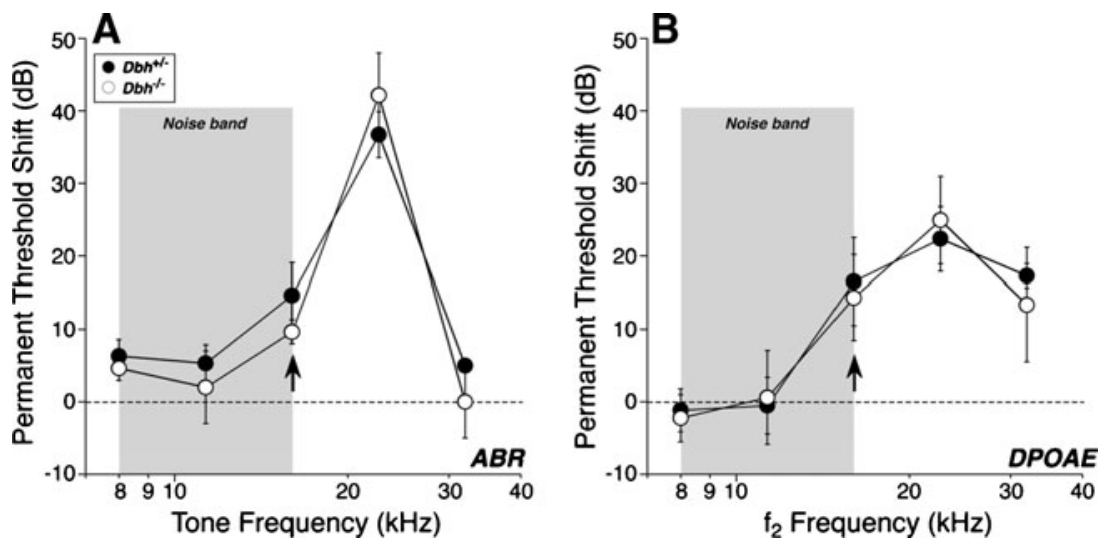

FIG. 7. Noise vulnerability is similar in $D b h^{-/-}$vs. $D b h^{+/-}$mice. A, B Mean permanent threshold shifts $( \pm$ SEM $)$ measured by ABRs (A) or DPOAEs (B) 1 week after exposure to an $8-16 \mathrm{kHz}$ noise band (gray bars) at $100 \mathrm{~dB}$ SPL for $2 \mathrm{~h}$. There were $12 \mathrm{Dbh}^{+/-}$and $6 \mathrm{Dbh}^{-/-}$ ears in the DPOAE measurement groups and half that many for the ABRs.

in the $D b h^{-/-}$mice with no externally visible signs of middle ear infection (Fig. 4B). The observation that DPOAE thresholds were more affected than ABR thresholds (Fig. 4A vs. B) is consistent with presence of a conductive hearing loss, since the OAE response amplitude is attenuated twice, by an acoustic round trip through the middle ear (Qin et al. 2010). The frequency trend of the hearing loss is also consistent, since the replacement of middle-ear air by fluid and/ or the slightly negative middle-ear pressures often associated with a non-patent Eustachian tube, will affect transmission of low frequencies more than high frequencies (Ravicz et al. 2004; Gan et al. 2006). A possible role of sympathetic innervation in the control of bone remodeling in the bulla (Sherman and Chole 2001) must also be considered in the etiology of the conductive hearing loss in the $D b h^{-/-}$mice. Regardless of the precise etiology, the present results add strong support to the idea that adrenergic signaling is important in the maintenance of a healthy middle ear.

Normal cochlear function does not require adrenergic signaling

Anatomical studies have documented a profuse sympathetic innervation from the stellate ganglion of the major vessels providing the cochlea's blood supply, i.e., the vertebral, basilar, anterior cerebellar, and labyrinthine arteries (Spoendlin and Lichtensteiger 1966), as well as a substantial population of adrenergic fibers from the superior cervical ganglion projecting to regions of the inner ear where the peripheral axons and cell bodies of the cochlear nerve are found (Hozawa et al. 1989; Shibamori et al. 1994; Spoendlin and Lichtensteiger 1967 and Fig. 1). In addition, adrenergic effects on trans-epithelial ion transport by the stria vascularis (Schimanski et al. 2001), a tissue with no direct sympathetic innervation, also suggest a role for humoral adrenaline on cochlea function. Despite this extensive evidence for adrenergic signal- ing in the cochlea, we found no abnormalities in the thresholds or suprathreshold responses of the $D b h^{-/-}$ ears by either DPOAE or ABR metrics, once ears with middle-ear infections were removed from consideration (Fig. 4).

Physiological studies have documented reductions in cochlear blood flow with electrical stimulation of the stellate ganglion (Laurikainen et al. 1993; Ren et al. 1993a) as well as increases in blood flow after its transection (Laurikainen et al. 1997). The ABR and DPOAE metrics should be sensitive indicators of cochlear blood flow: DPOAE and ABR amplitudes have been shown to decrease within 15 and $30 \mathrm{~s}$, respectively, of compression of the internal auditory artery (Telischi et al. 1999). ABR and DPOAE should also be sensitive indicators of changes in the endolymphatic potential (Rubsamen et al. 1995), which is intimately dependent on the $\mathrm{K}^{+}$transport activity of the stria. Animal studies suggest that neural thresholds rise $1 \mathrm{~dB}$ for every $\mathrm{mV}$ decrease in the EP (Sewell 1984). The ABR and DPOAE data in Figure 4 strongly suggest that both cochlear blood flow and the endolymphatic potential are normal in the $D b h^{-/-}$ mice, despite the complete and chronic lack of adrenergic signaling.

Physiological studies have also documented changes in the amplitude (Pickles 1979) or latency (Lee and Møller 1985) of cochlear neural responses during electrical stimulation of the superior cervical ganglion, without changes in hair cell potentials (i.e., cochlear microphonic: Pickles 1979), consistent with a post-synaptic effect of the "free" endings of sympathetic fibers on cochlear neurons in the region of the habenula perforata (Fig. 1). In contrast, we saw no evidence for chronic effects on the excitability of the auditory nerve in $D b h^{-/-}$mice (Fig. 4). The sensitivity of ABR amplitudes as a measure of neural excitability has been demonstrated in mice lacking CGRP, a neurotransmitter in the lateral olivocochlear efferents projecting post-synaptically to cochlear neurons: we showed that a decrease in ABR amplitudes of as little 

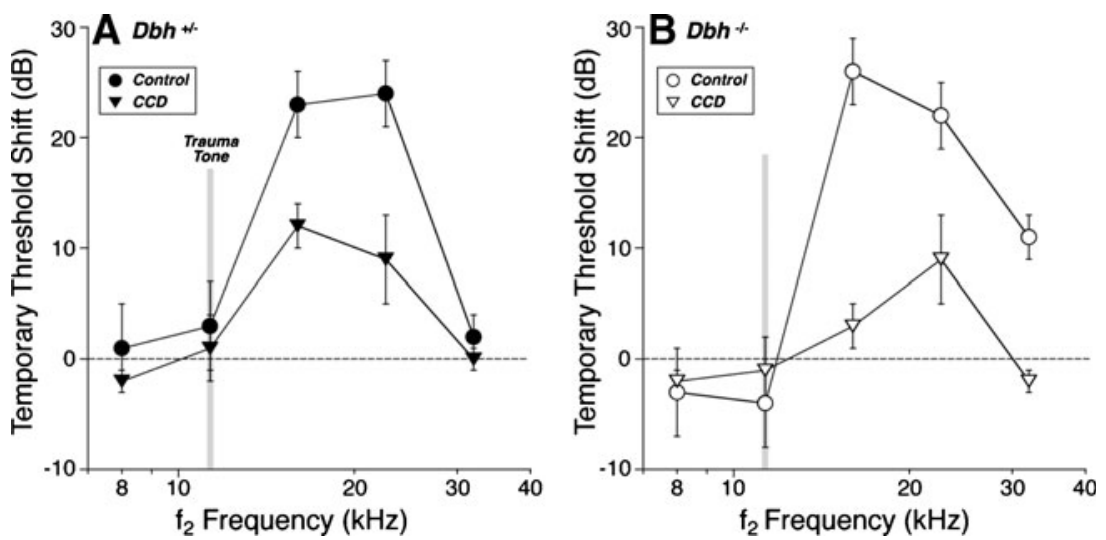

FIG. 8. Protective effect of contralateral cochlear destruction (CCD) on ipsilateral noise-induced threshold shift is not extinguished by removal of adrenergic signaling. Mean DPOAE threshold shifts $( \pm$ SEM) were recorded 5-10 min after exposure to an 11$\mathrm{kHz}$ pure tone (gray bar) at $102 \mathrm{~dB} \mathrm{SPL}$ for $1 \mathrm{~min}$ in groups of $D b h^{+-}$(A $n=11$ animals) and $\mathrm{Dbh}^{-/-}$(B $n=5$ animals) (circles). In a second experiment (triangles), groups of $\operatorname{Dbh}^{+-}(\mathbf{A} n=5)$ and $D b h^{-/-}(\mathbf{B} n=7)$ animals underwent a contralateral cochlear destruction $24 \mathrm{~h}$ prior to the trauma-tone exposure. Protective effects of this surgical manipulation were similar in both genotypes. as $20 \%$ was statistically significant, with no change in DPOAE responses (Maison et al. 2003b). In reconciling these superficially conflicting results, it is important to note (1) that the sympathetic innervation remains intact in the $D b h^{-/-}$mice (Jin et al. 2004), and (2) that cochlear sympathetic fibers may release a number of other neurotransmitters besides noradrenaline (Sanchez-Garzon et al. 1990). Thus, effects reported to arise from electrical stimulation of the sympathetic ganglia may be mediated by nonadrenergic signaling pathways in the fibers they give rise to.

\section{Adrenergic signaling and vulnerability to acoustic injury}

Numerous investigators have wondered if a functional role for the cochlea's sympathetic nervous system might be revealed by stressing the ear with overexposure to sound and looking for an effect of surgical removal of the superior cervical ganglion on the resultant threshold elevation (e.g., Bielefeld and Henderson 2007; Borg 1982; Hildesheimer et al. 2002; Horner et al. 2001). All such studies finding any effect concluded that loss of the adrenergic innervation protected the ear from acoustic injury, i.e., that noiseinduced threshold shifts were smaller after sympathectomy by 10-15 dB (Borg 1982; Giraudet et al. 2002; Hildesheimer et al. 1991; Hildesheimer et al. 2002; Horner et al. 2001). The explanations proposed for this surprising result were either (1) that vesselassociated sympathetics normally cause noise- or stress-induced cochlear vasoconstriction that exacerbates the noise-induced damage (e.g., Hildesheimer et al. 1991), or (2) that the vessel-independent sympathetics projecting to cochlear nerve fibers exacerbate the damage by some unknown mechanism (e.g., Horner et al. 2001). The first interpretation is clouded by the facts that the superior cervical ganglion does not give rise to the vessel-associated cochlear fibers (Densert 1974; Hozawa et al. 1989;
Spoendlin 1981) and that its electrical stimulation does not change cochlear blood flow (Ren et al. 1993b). Both interpretations are complicated by the fact that several studies report bilateral changes in noise vulnerability from unilateral sympathectomy, despite the strictly unilateral projection from the superior cervical ganglion (Giraudet et al. 2002; Horner et al. 2001), which is very difficult to explain.

In the present study, we saw no effect of adrenergic signaling on the response of the cochlea to acoustic overstimulation, despite the fact our noise-exposure paradigm (spectra, levels, and durations) were similar to those in the other studies: noise-induced permanent (Fig. 7) and temporary (Fig. 8) threshold shifts were identical in $D b h^{-/-}$ mice and their littermate controls. There are at least three ways to resolve this apparently paradoxical result. Firstly, as noted above, the loss of catecholamines (in the present study) is not the same as the loss of sympathetic innervation (in the other studies): the anti-protective effect seen after sympathectomy could arise from absence of a neuropeptide colocalized with noradrenaline in the sympathetic terminals (Sanchez-Garzon et al. 1990), which could well be unaffected in the present study. Secondly, the sympathectomy could have caused a subtle conductive hearing loss (as seen in Fig. 4B), reducing the effective sound level and thus the threshold shift. The relation between exposure level and threshold shift is highly non-linear such that a threshold shift decrease of $\sim 10-15 \mathrm{~dB}$ requires only a $2-3 \mathrm{~dB}$ decrease in the effective exposure level (Yoshida et al. 2000): such a small conductive loss would easily go undetected. Thirdly, the strength of shock-evoked olivocochlear effects was enhanced in the $D b h^{-1-}$ mice (Fig. 6), and this type of olivocochlear activation can protect the ear from acoustic injury, as shown in a transgenic mouse overexpressing the $\alpha 9$ acetylcholine receptor (Maison et al. 2002), which showed enhancement of shock-evoked olivocochlear effects coupled with increased resistance to intense noise. Thus, a 
protective effect of enhanced olivocochlear activity might have masked an anti-protective effect of removal of the sympathetic function in the $D b h^{-/-}$ mice.

A change in shock-evoked olivocochlear effects in $\mathrm{Dbh}^{-1-}$ (Fig. 6B) is consistent with studies showing (1) noradrenergic terminals (from the locus coeruleus) on the cell bodies of medial olivocochlear neurons (Mulders and Robertson 2005a; Thompson and Schofield 2000; Woods and Azeredo 1999) and (2) largely excitatory effects of catecholamines on olivocochlear cells in slice preparations (Wang and Robertson 1997). In our olivocochlear assay, the size of efferent effects, i.e., the change in DPOAE amplitudes, is proportional to the change in discharge rates in olivocochlear fibers elicited by the shocks. Since there is both spontaneous and sound-evoked discharge in OC fibers in the absence of shocks (Liberman 1988), a reduction in these "baseline" rates, due to loss of adrenergic signaling, could enhance the efferent effects observed in our assay when shocking the olivocochlear bundle. Thus, present results support a role for central adrenergic signaling in modulating olivocochlear feedback to the inner ear.

The notion of central adrenergic modulation of the olivocochlear pathway suggested that protection of the ipsilateral ear from acoustic injury by prior destruction or lignocaine-silencing of the contralateral ear (Rajan and Johnstone 1989; Robertson and Anderson 1994) might arise via a stress-related activation of the locus coeruleus and subsequent transient enhancement of the sound-evoked olivocochlear feedback pathway. Prior work has shown that this protection requires the olivocochlear bundle (Rajan and Johnstone 1989); present results show definitively that adrenergic signaling is not required for the putative enhancement of olivocochlear feedback to the ear.

In summary, the present results show that inner ear function is surprisingly normal in the complete absence of adrenergic signaling. It is possible that a more dramatic phenotype would appear if catecholamines were suddenly removed in adulthood, rather than eliminated neonatally, as in the present study. Alternatively, it is possible that the functional significance of this homeostatic system is not revealed in the absence of the appropriate environmental stressors, such as immunological challenges to the inner ear, which are absent in captivity within an institutional animal care facility.

\section{ACKNOWLEDGEMENTS}

This work was supported by NIH Grants: R01 DC0188 (MCL), DC000194 (JJR), MH063352 (SAT) and P30 DC5029 (MCL). The skillful assistance of Leslie Dodds, Connie Miller and Melissa Woods is gratefully acknowledged.

\section{REFERENCES}

Alaniz RC, Thomas SA, Perez-Melgosa M, Mueller K, Farr AG, PAlmiter RD, Wilson CB (1999) Dopamine beta-hydroxylase deficiency impairs cellular immunity. Proc Natl Acad Sci USA 96:2274-2278

Bellinger Dl, Millar ba, Perez S, Carter J, Wood C, Thyagarajan S, Molinaro C, Lubahn C, Lorton D (2008) Sympathetic modulation of immunity: relevance to disease. Cell Immunol 252:2756

Bielefeld EC, Henderson D (2007) Influence of sympathetic fibers on noise-induced hearing loss in the chinchilla. Hear Res 223:11-19

Borg E, Densert O, Flock A (1974) Synaptic vesicles in the cochlea. Acta Otolaryngol 78:321-332

Borg E (1982) Protective value of sympathectomy of the ear in noise. Acta Physiol Scand 115:281-282

Darrow KN, Simons EJ, Dodds L, Liberman MC (2006) Dopaminergic innervation of the mouse inner ear: evidence for a separate cytochemical group of cochlear efferent fibers. J Comp Neurol 498:403-414

Densert O (1974) Adrenergic innervation in the rabbit cochlea. Acta Otolaryngol 78:345-356

Densert O, Flock A (1974) An electron-microscopic study of adrenergic innervation in the cochlea. Acta Otolaryngol 77:185-197

Fauser C, Schimanski S, Wangemann P (2004) Localization of beta1adrenergic receptors in the cochlea and the vestibular labyrinth. J Membr Biol 201:25-32

GAN RZ, DAI C, WoOD WW (2006) Laser interferometry measurments of middle ear fluid and pressure effects on sound transmission. J Acoust Soc Am 120:3799-3810

Giraudet F, Horner KC, Cazals Y (2002) Similar half-octave TTS protection of the cochlea by xylazine/ketamine or sympathectomy. Hear Res 174:239-248

Guinan JJ (1996) The physiology of olivocochlear efferents. In: Dallos PJ, Popper AN, Fay RR (eds) The Springer Verlag handbook of auditory research: the cochlea, Vol. VIII. Springer Verlag, New York

Hequembourg S, Liberman MC (2001) Spiral ligament pathology: a major aspect of age-related cochlear degeneration in C57BL/6 mice. J Assoc Res Otolaryngol 2:118-129

Hildesheimer M, Sharon R, Muchnik C, Sahartov E, Rubinstein M (1991) The effect of bilateral sympathectomy on noise induced temporary threshold shift. Hear Res 51:49-53

Hildesheimer M, Henkin Y, Pye A, Heled S, Sahartov E, Shabtai El, MuchNIк C (2002) Bilateral superior cervical sympathectomy and noise-induced, permanent threshold shift in guinea pigs. Hear Res $163: 46-52$

Hori T, Katafuchi T, Take S, Shimizu N, Nijima A (1995) The autonomic nervous system as a communication channel between the brain and the immune system. Neuroimmunomodulation 2:203-215

Horner KC, Giraudet F, Lucciano M, Cazals Y (2001) Sympathectomy improves the ear's resistance to acoustic trauma-could stress render the ear more sensitive? Eur J Neurosci 13:405-408

Hozawa J, Kimura RS, Takahashi S (1989) Sympathetic nervous system in the guinea pig cochlea. Ear Res Jpn 20:111-112

HozaWa K, Kimura RS (1990) Cholinergic and noradrenergic nervous systems in the cynomolgus monkey cochlea. Acta Otolaryngol 110:46-55

Jin SH, Kim HJ, Harris DC, Thomas SA (2004) Postnatal development of the cerebellum and the CNS adrenergic system is independent of norepinephrine and epinephrine. J Comp Neurol 477:300-309

Laurikainen EA, Kim D, Didier A, Ren T, Miller JM, Quirk WS, NutTall AL (1993) Stellate ganglion drives sympathetic regulation of cochlear blood flow. Hear Res 64:199-204 
Laurikainen EA, Ren T, Miller JM, Nuttall AL, Quirk WS (1997) The tonic sympathetic input to the cochlear vasculature in guinea pig. Hear Res 105:141-145

Lee AH, MølLer AR (1985) Effects of sympathetic stimulation on the round window compound action potential in the rat. Hear Res 19:127-134

Li Z, Oben JA, Yang S, Lin H, Stafford EA, Soloski MJ, Thomas SA, DieHL AM (2004) Norepinephrine regulates hepatic innate immune system in leptin-deficient mice with nonalcoholic steatohepatitis. Hepatology 40:434-441

Liberman MC (1988) Response properties of cochlear efferent neurons: monaural vs. binaural stimulation and the effects of noise. J Neurophysiol 60:1779-1798

Maison SF, Luebke AE, Liberman MC, Zuo J (2002) Efferent protection from acoustic injury is mediated via alpha9 nicotinic acetylcholine receptors on outer hair cells. J Neurosci 22:10838-10846

Maison SF, Adams JC, Liberman MC (2003A) Olivocochlear innervation in the mouse: immunocytochemical maps, crossed versus uncrossed contributions, and transmitter colocalization. J Comp Neurol 455:406-416

Maison SF, Emeson RB, Adams JC, Luebke AE, Liberman MC (2003B) Loss of alpha CGRP reduces sound-evoked activity in the cochlear nerve. J Neurophysiol 90:2941-2949

Maison SF, VetTer DE, Liberman MC (2007) A novel effect of cochlear efferents: in vivo response enhancement does not require alpha9 cholinergic receptors. J Neurophysiol 97:3269-3278

Molina PE (2005) Neurobiology of the stress response: contribution of the sympathetic nervous system to the neuroimmune axis in traumatic injury. Shock 24:3-10

Mulders WH, Robertson D (2005A) Catecholaminergic innervation of guinea pig superior olivary complex. J Chem Neuroanat 30:230-42

Mulders WH, Robertson D (2005в) Noradrenergic modulation of brainstem nuclei alters cochlear neural output. Hear Res 204:147-55

Nagaraj BS, Linthicum FH JR (1998) Autonomic innervation of the human middle ear: an immunohistochemical study. Am J Otolaryngol 19:75-82

NANCE DM, SANDERS VM (2007) Autonomic innervation and regulation of the immune system (1987-2007). Brain Behav Immun 21:736-745

PickLes JO (1979) An investigation of sympathetic effects on hearing. Acta Otolaryngol 87:69-71

QIN Z, Wood M, Rosowski JJ (2010) Measurement of conductive hearing loss in mice. Hear Res 263:93-103

Rajan R, Johnstone BM (1989) Contralateral cochlear destruction mediates protection from monoaural loud sound exposures through the crossed olivocochlear bundle. Hear Res 39:263-277

Ravicz ME, Rosowski JJ, Merchant SN (2004) Mechanisms of hearing loss resulting from middle-ear fluid. Hear Res 195:103-130

Ren TY, Laurikainen E, Quirk WS, Miller JM, Nuttall AL (1993A) Effects of stellate ganglion stimulation on bilateral cochlear blood flow. Ann Otol Rhinol Laryngol 102:378-384

Ren TY, Laurikainen E, Quirk WS, Miller JM, Nuttall AL (1993B) Effects of electrical stimulation of the superior cervical ganglion on cochlear blood flow in guinea pig. Acta Otolaryngol 113:146-151

Robertson D, Anderson CJ (1994) Acute and chronic effects of unilateral elimination of auditory nerve activity on susceptibility to temporary deafness induced by loud sound in the guinea pig. Brain Res 646:37-43

Rosowski JJ, Brinsko KM, Tempel BI, KujaWa SG (2003) The aging of the middle ear in 129S6/SvEvTac and CBA/CaJ mice: measurements of umbo velocity, hearing function, and the incidence of pathology. JARO 4:371-383

Rubsamen R, Mills DM, Rubel EW (1995) Effects of furosemide on distortion product otoacoustic emissions and on neuronal responses in the anteroventral cochlear nucleus. J Neurophysiol 74:1628-1638

Sanchez-Garzon M, Tramu G, Tillet Y, Pontarotti P, Eybalin M (1990) Neuropeptides in the sympathetic innervations of the cochlea. Adv Otorhinolaryngol 45:99-102

Schimanski S, Scofield MA, Wangemann P (2001) Functional beta2adrenergic receptors are present in nonstrial tissues of the lateral wall in the gerbil cochlea. Audiol Neurootol 6:124-131

SEwell WF (1984) The effects of furosemide on the endocochlear potential and auditory-nerve fiber tuning curves in cats. Hear Res 14:305-314

Sherman BE, Chole RA (2001) Effect of chemical sympathectomy with 6-hydroxydopamine on osteoclast activity in the gerbilline middle ear bulla. Otol Neurotol 22:237-241

Shibamori Y, TAMAMaki N, Satto H, Nojyo Y (1994) The trajectory of the sympathetic nerve fibers to the rat cochlea as revealed by anterograde and retrograde WGA-HRP tracing. Brain Res 646:223-229

Spoendlin H (1981) Autonomic innervation of the inner ear. Adv Otorhinolaryngol 27:1-13

Spoendin H, Lichtensteiger W (1966) The adrenergic innervation of the labyrinth. Acta Otolaryngol 61:423-434

Spoendin H, Lichtensteiger W (1967) The sympathetic nerve supply to the inner ear. Archiv klin exper Ohren-Nasen- und Kehlkopfheilk 189:346-359

Straub RH, Pongratz G, Weidler C, Linde HJ, Kirschning CJ, Gluck T, Scholmerich J, FAlk W (2005) Ablation of the sympathetic nervous system decreases gram-negative and increases gram-positive bacterial dissemination: key roles for tumor necrosis factor/phagocytes and interleukin-4/lymphocytes. J Infect Dis 192:560-572

Svane-Knudsen V, Kruse S, Lildholdt T, Madsen T (1986) Sympathetic influence on the normal Eustachian tube. An experimental study in the rat. Acta Otolaryngol 101:263-268

Telischi FF, Mom T, Agrama M, Stagner BB, Ozdamar O, Bustillo A, MARTIN GK (1999) Comparison of the auditory-evoked brainstem response wave I to distortion-product otoacoustic emissions resulting from changes to inner ear blood flow. Laryngoscope 109:186-191

Templeton A, Nguyen G, Ash JD, Straub RH, Carr DJ (2008) Chemical sympathectomy increases susceptibility to ocular herpes simplex virus type 1 infection. J Neuroimmunol 197:3746

Thomas SA, PALmiter RD (1997A) Impaired maternal behavior in mice lacking norepinephrine and epinephrine. Cell 91:583-592

Thomas SA, Palmiter RD (1997в) Disruption of the dopamine betahydroxylase gene in mice suggests roles for norepinephrine in motor function, learning, and memory. Behav Neurosci 111:579-589

Thomas SA, Palmiter RD (1997c) Thermoregulatory and metabolic phenotypes of mice lacking noradrenaline and adrenaline. Nature 387:94-97

Thomas SA, Matsumoto AM, Palmiter RD (1995) Noradrenaline is essential for mouse fetal development. Nature 374:643-646

Thomas SA, Marck BT, Palmiter RD, Matsumoto AM (1998) Restoration of norepinephrine and reversal of phenotypes in mice lacking dopamine beta-hydroxylase. J Neurochem 70:2468-2476

Thompson AM, Schofield BR (2000) Afferent projections of the superior olivary complex. Microsc Res Tech 51:330-354

Uddman R, Kitajiri M, Sundler F (1983) Autonomic innervation of the middle ear. Ann Otol Rhinol Laryngol 92:151-154

Wada T, Takahashi K, Ito Z, Hara A, Takahashi H, Kusakari J (1999) The protective effect of the sympathetic nervous system against acoustic truama. Auris Nasus Larynx 26:375-382

WANG X, RoberTson D (1997) Two types of actions of norepinephrine on identified auditory efferent neurons in rat brain stem slices. J Neurophysiol 78:1800-1810 
Wang HW, Lin JK, Wang JY (1994) Sympathetic innervation of the eustachian tube in rats. Eur Arch Otorhinolaryngol 251:283-286

Wang Y, Hirose K, Liberman MC (2002) Dynamics of noise-induced cellular injury and repair in the mouse cochlea. JARO 3:248-268

Wiederhold ML, Kiang NY (1970) Effects of electric stimulation of the crossed olivocochlear bundle on single auditory-nerve fibers in the cat. J Acoust Soc Am 48:950-965
Woods CI, Azeredo WJ (1999) Noradrenergic and serotonergic projections to the superior olive: potential for modulation of olivocochlear neurons. Brain Res 836:9-18

Yoshida N, Hequembourg SJ, Atencio CA, Rosowski JJ, Liberman MC (2000) Acoustic injury in mice: 129/SvEv is exceptionally resistant to noise-induced hearing loss. Hear Res 141: $97-106$ 